\title{
Post Locoregional Therapy Treatment Imaging in Hepatocellular Carcinoma Patients: A Literature-based Review
}

\author{
Shamar Young, Andrew J. Taylor* and Tina Sanghvi \\ Department of Radiology, University of Minnesota, Minneapolis, MN, USA
}

\begin{abstract}
Imaging plays a crucial role in the diagnosis of hepatocellular carcinoma (HCC) as well as in determining treatment efficacy, or complications, following therapy. Unlike other cancers, HCC is most commonly treated by locoregional therapies (LRTs) such as thermal ablation, transarterial chemoembolization, and transarterial radioembolization. These treatments can lead to changes on imaging that make determination of residual/ recurrent disease difficult. This literature-based review discusses the expected postimaging findings following LRT.

Citation of this article: Young S, Taylor AJ, Sanghvi T. Post locoregional therapy treatment imaging in hepatocellular carcinoma patients: a literature-based review. J Clin Transl Hepatol 2018;6(2):189-197. doi: 10.14218/JCTH.2017.00059.
\end{abstract}

\section{Introduction}

Hepatocellular carcinoma (HCC) is the most common primary liver cancer and the third leading cause of cancer death in the world. ${ }^{1}$ Liver transplantation is the gold standard of treatment, however few patients are able to take advantage of this option due to social issues, the lack of available livers and/or the frequent late stage of disease at time of diagnosis. ${ }^{2}$ Furthermore, up to $25 \%$ of those patients meeting initial requirements and listed for transplantation will eventually drop off the transplant

Keywords: Hepatocellular carcinoma (HCC); Transarterial chemoembolization (TACE); Thermal ablation; Transarterial radioembolization (TARE); Magnetic resonance imaging (MRI).

Abbreviations: AASLD, American Association for the Study of Liver Diseases; $A D C$, apparent diffusion coefficient; $A P$, arterial phase; $C R$, complete response; $\mathrm{CT}$, computed tomography; CTACE, conventional TACE; DP, delayed phase; DWI, diffusion-weighted imaging; DEB TACE, drug-eluting bead TACE; DCE, dynamic contrast enhanced; EASL, European Association for the Study of the Liver; HCC, hepatocellular carcinoma; LAP, late arterial phase; LRT, locoregional therapies; LIRADS, liver imaging and reporting data system; MRI, magnetic resonance imaging; MWA, microwave ablation; mRECIST, modified Response Evaluation Criteria in Solid Tumors; NSF, nephrogenic systemic fibrosis; OPTN, Organ Procurement and Transplantation Network; PR, partial response; PVP, portal venous phase; $P D$, progressive disease; $q E A S L$, quantitative European Association for the Study of the Liver; RFA, radiofrequency ablation; RECIST, Response Evaluation Criteria in Solid Tumors; SD, stable disease; SIR, Society of Interventional Radiology; TACE, transarterial chemoembolization; TAE, transarterial embolization; TARE, transarterial radioembolization; US, ultrasound; UNOS, United Network for Organ Sharing; WHO, World Health Organization.

Received: 30 August 2017; Revised: 14 December 2017; Accepted: 22 December 2017

*Correspondence to: Andrew J. Taylor, Department of Radiology, University of Minnesota, 420 Delaware Street SE, MMC 292, Minneapolis, MN 55455, USA. Tel: +1-612-626-6638, Fax: +1-612-626-5505, E-mail: taylora@umn.edu list for a variety of reasons, including tumor progression. ${ }^{3}$ Surgical resection is considered to be curative, yet due to underlying liver disease and associated comorbidities few patients are considered surgical candidates. Surgery, despite improved techniques, also confers a higher than desirable mortality and morbidity ${ }^{4}$ and, in spite of these risks, recurrence rates remain significant, reported to be as much as $50 \%$ at 2 years. ${ }^{5}$ The only systemic therapy in regular use, sorafenib, has been shown to have a very modest (2.3-2.8 months) survival advantage in patients with advanced HCC.6,7 Given the modest survival advantage and side effect profile, sorafenib is typically reserved for patients with advanced disease who are not candidates for other therapies.

Locoregional therapies (LRTs) are therefore the most common method utilized to treat HCC, and can be subdivided into thermal ablation and intra-arterial therapies. Thermal ablation is notable as the only LRT considered curative in well-chosen patients, being equivalent to surgical resection. ${ }^{8,9}$ Intra-arterial therapies include transarterial embolization (TAE), transarterial chemoembolization (TACE), and transarterial radioembolization (TARE). These latter therapies are considered life prolonging, but ultimately palliative. Intraarterial therapies are also frequently used in a "bridging" fashion to either get patients within transplant or Milan criteria, or to keep them within criteria while they are on the transplant list.

Several societies have developed guidelines to help standardize criteria for both the imaging diagnosis and assessment of treatment response in $\mathrm{HCC}$, including Organ Procurement and Transplantation Network (OPTN)/United Network for Organ Sharing (UNOS), American Association for the Study of Liver Diseases (AASLD), Liver Imaging and Reporting Data System (LI-RADS), and the European Association for the Study of the Liver (EASL). ${ }^{10-13}$ HCC is one of the few cancers where imaging appearance alone, without pathology, can be used to diagnose disease. Similarly, imaging is used to diagnose residual or recurrent disease following treatment with LRTs. Unfortunately, given the iatrogenically-induced changes that can be associated with LRTs, determining treatment effect versus residual tumor can be difficult.

This paper uses the latest literature to review the imaging findings resulting from the various LRTs.

Response classification systems

Response classification systems have been developed to standardize terminology, assist in research standardization, and improve interdisciplinary dialogue. Initial classification systems included the Response Evaluation Criteria in Solid 
Young S. et al: Imaging review of post LRTs in HCC

Tumors (RECIST) and World Health Organization (WHO) guidelines that focused on the overall size of the lesion based upon the sum of diameters, using one (RECIST) or two axial dimensions (WHO). ${ }^{14-16}$ These systems, which were designed primarily for cytotoxic, systemic therapy, fell short in evaluating the response following LRT.

The WHO and RECIST criteria revolve around measuring the size of the lesion without accounting for the cellular makeup or viability of that lesion. Because LRTs induce necrosis, through various methods, WHO and RECIST criteria inadequately report the change in tumor viability. Using these criteria can lead to apparent "tumor progression", as the postLRT treatment zone is frequently larger than the initial tumor dimensions.

Because of these limitations two new response classification systems were developed, the modified Response Evaluation Criteria in Solid Tumors (mRECIST) and EASL guidelines. ${ }^{13,17}$ These criteria use contrast-enhanced computed tomography (CT) or magnetic resonance imaging (MRI) to assess response by using arterial enhancement as a marker for residual, viable tumor and the lack of enhancement as a marker for necrosis. Enhancement is critical in postLRT imaging because the appearance of necrosis is variable and unreliable on noncontrast enhanced imaging. ${ }^{18,19}$ These systems measure the maximal diameter of the residual tumor (that is, the arterial enhancing portion) in one (mRECIST) or two (EASL) axial dimensions and classify the effect to treatment as complete response (CR), partial response (PR), stable disease (SD), or progressive disease (PD). The definition of each response category by classification system can be found in Table 1 .

These enhancement-based criteria (mRECIST and EASL) have been shown to be more accurate predictors of response and survival than the "size alone" criteria (WHO and RECIST) on radiological-pathologic correlation studies. ${ }^{20,21}$ Even so, these systems, on occasion, also incur trouble differentiating benign posttreatment findings from residual/recurrent disease, for example, when patchy arterial enhancement is interspersed within areas of hypoenhancement. ${ }^{22}$

Continued interest in improvement and refinement of the response classification systems has led to the recent development of quantitative EASL ( $\mathrm{qEASL}$ ) criteria. This system was developed to address certain deficiencies in the abovediscussed response criteria relating to pathologic correlation and interreader agreement. ${ }^{23,24}$ The qEASL criteria utilize volumetric measurements to gauge residual/recurrent disease extent compared with the mono or bidirectional axial measurements of mRECIST and EASL respectively. ${ }^{23,24}$

These volumetric measurements will both better represent the residual posttreatment tumor and possibly help differentiate responders versus nonresponders in a timelier manner. ${ }^{24,25}$
The principal drawback of this method is the increased labor and time required for volumetric measurements. However, rapid software development using fully or semiautomatic segmentation to provide this $3 \mathrm{D}$ measurement is making the delivery of this data more workflow friendly. ${ }^{25}$

\section{Imaging modality for LRT follow-up}

The most commonly used modalities to evaluate treatment following LRTs are contrast-enhanced multiphase MRI and CT. In comparison analyses among CT, contrast-enhanced ultrasound (US) and MRI, MRI was found to be the most sensitive and specific modality in determining the presence or absence of residual tumor following TACE. ${ }^{26}$ The use of CT or MRI, however, varies by institution. Notably, CT has a reduced associated cost, requires less patient cooperation, and suffers less degradation when ascites is present. ${ }^{27}$

As discussed above, intravenous contrast is necessary for these evaluations, as posttreatment lesion size alone is not an accurate predictor of treatment response. Given the risk of nephrogenic systemic fibrosis (NSF) associated with administering gadolinium-based contrast agents in patients with impaired renal function, CT follow-up may be preferred in some cases. Contrast-enhanced US has been investigated, but has not performed as well as either CT or MRI and is therefore rarely used. ${ }^{26}$

\section{Technique of multiphase enhancement MRI}

Ideally, MRI is a better choice for HCC diagnosis and characterization compared with CT. MRI has both better contrast delineation between the HCC and liver as well as more sequences to dissect out a HCC from the underlying liver leading to MRI's higher sensitivity than CT with nearly $100 \%$ specificity. ${ }^{28}$ It is beyond the scope of this paper to review in detail the components of an MRI exam and interpretation methods for HCC detection/characterization. But, a basic MRI exam consists of standard sequences which include T2-weighted imaging both with and without fat suppression, T1-weighted in- and opposed-phase sequences, diffusion-weighted imaging (DWI), and unenhanced followed by dynamic contrast-enhanced (DCE) $3 \mathrm{D}$ gradient-recalled echo fat-suppressed imaging using either gadolinium-based extracellular or hepatobiliary agents.

DCE images typically consist of an arterial phase (AP), portal venous phase (PVP) (65-85 seconds from the beginning of injection), and a delayed phase (DP) (3-5 minutes from beginning of injection). The optimal AP is the "late arterial phase" (LAP) defined as contrast visualized in the hepatic artery, portal vein, but not yet in the hepatic veins. This allows time not only for the contrast agent to be delivered to the

Table 1. Response criteria categories

\begin{tabular}{llll}
\hline Response & $\begin{array}{l}\text { mRECIST: Unidirectional } \\
\text { Ax measurement }\end{array}$ & $\begin{array}{l}\text { EASL: Bidirectional } \\
\text { Ax measurement }\end{array}$ & $\begin{array}{l}\text { qEASL: Volumetric } \\
\text { measurement }\end{array}$ \\
\hline Progressive Disease (PD) & $\begin{array}{l}20 \% \text { increase in size } \\
\text { or new lesion }\end{array}$ & $\begin{array}{l}25 \% \text { increase in size } \\
\text { or new lesion }\end{array}$ & $\begin{array}{l}\text { 25\% increase in size } \\
\text { or new lesion }\end{array}$ \\
Stable Disease (SD) & Neither PR nor PD criteria met & Neither PR nor PD & Neither PR nor PD \\
Partial Response (PR) & $30 \%$ decrease in size & $50 \%$ decrease in size & $50 \%$ decrease in size \\
Complete Response (CR) & No residual area of disease & No residual area of disease & No residual area of disease
\end{tabular}

Abbreviations: Ax, axial; EASL, European association for the study of the liver; mRECIST, modified response evaluation criteria in solid tumors; qEASL, quantitative EASL. 
arterial fed tumor but also for the contrast agent to proceed into the tumor interstitium, allowing the tumor to enhance relative to the surrounding liver parenchyma. A HCC will typically have hyperenhancement during the LAP with "washout" (the tumor's lower signal intensity compared with the adjacent liver) during the PVP and/or DP to meet major criteria for HCC imaging diagnosis, either at initial diagnosis or as evidence of residual/recurrence postLRT. The other sequences in this exam each have a role in HCC detection/characterization, but this is again beyond this article's scope. It is important to note, subtraction imaging can be helpful given the inherent increased T1 signal intensity in treated lesions. ${ }^{29,30}$

\section{Technique of multiphase enhancement CT}

While CT has been shown to be less effective in HCC detection and characterization than MRI, CT does have qualities that can make it an exam of choice: it takes less patient cooperation, is less affected by large volume ascites, is potentially safer in patients with renal failure, is more readily available, and is cheaper than MRI.

The optimal multidetector, multiphasic CT consists of four separate phases through the liver: noncontrast, LAP, PVP and DP, with timing that mirrors that of MRI. While MRI has other criteria available to help in HCC display, CT has mainly the various phases of contrast enhancement. The AP hyperenhancement and washout of the PVP and/or DP will be used to assess for HCC in both pre- or posttreatment.

\section{LRT techniques and posttreatment imaging appearances}

Each LRT has a different method of inducing tumor death. These unique methods of inducing tumor death can lead to differences in the characteristics of posttreatment imaging. The following sections will briefly discuss each technique followed by its important posttreatment imaging characteristics.

\section{Ablation}

\section{Technique}

"Ablation" is a general term used to refer to various techniques that use the insertion of probes or needles into a tumor to deliver tumoricidal treatment. Current techniques use thermal ablation to treat HCC. Thermal ablation includes modalities that use cold (cryoablation) and heat (either microwave ablation (MWA) or radiofrequency ablation (RFA)) to induce tumor death. Because cryoablation has been shown to induce an immune reaction leading to fever, thrombocytopenia and/or shock (termed "cryoshock") when treating liver lesions its use has fallen out of favor in current practice. ${ }^{31}$

RFA and MWA are currently the most frequently utilized ablation techniques for HCC. Both use heat to induce coagulative necrosis thus tumor death. They vary both in the method used to induce cell death and the size of ablation zone they potentially create; however, these technical differences are inconsequential in terms of posttreatment imaging. The technique consists of placing one or more needle-shaped probes into the lesion, which then generate an area of lethal temperature around them. Next, the probes are retracted, whilst still producing heat to "ablate" the tract.

Other ablative techniques, such as irreversible electroporation, ethanol ablation and chemical ablation, have been reported, ${ }^{32,33}$ but these modalities are rarely used, and do not warrant further discussion at this time.

Contraindications for ablation is a complex subject, which is dynamically changing as technical improvement occurs, and new treatment algorithms are developed. In general, the contraindications for its use are lesions which are large in size, located adjacent to a major vessel and/or located near a critical structure (major bile duct, gall bladder, diaphragm, colon, stomach).

\section{Imaging after ablation}

Assessing imaging changes by size alone correlates poorly with treatment effect. As ablation of an extra $0.5-1 \mathrm{~cm}$ rim of normal liver around the tumor is necessary for adequate treatment of potential microscopic tumor extension, the treated target area should be larger than the original tumor following ablation. ${ }^{34,35}$ In addition, the ablation-induced necrosis, edema and inflammation in and of itself will often lead to an increase in size initially. ${ }^{36,37}$ The zone of ablation typically decreases in size over the ensuing months.

If imaging is done within the first several days following ablation, gas bubbles are frequently seen, representing posttreatment hydrogen gas, and should be of no concern (Fig. 1). These bubbles, not indicative of infection, normally resolve within the first couple of weeks. A more troublesome finding/complication following treatment is the presence of active hemorrhage (Fig. 2).

A common postablation finding is a hypervascular rim surrounding the ablation zone that can remain for several months. This hypervascular rim is initially secondary to hyperemia and then inflammation resulting from the ablation process. ${ }^{38}$ The imaging manifestation of this hypervascular rim is seen during arterial enhancement. One study demonstrated this rim in $89 \%$ of cases at 1 month, $56 \%$ at $1-3$ months, and $22 \%$ at 3-6 months imaging. ${ }^{37}$ The fact that the vast majority of recurrences also occur along the treatment zone periphery may result in some confusion discerning expected marginal enhancement from residual or recurrent tumor. ${ }^{35,39}$

This expected posttreatment rim enhancement should be $\leq 5 \mathrm{~mm}$, which may continue into the PVP (Fig. $3 \mathrm{~A}$ and $3 \mathrm{~B}$ ). Conversely, residual/recurrent disease along the periphery is typically associated with $>5 \mathrm{~mm}$ area of AP enhancement and/ or washout on PVP/DP imaging (Fig. 3C and 3D). ${ }^{38,40}$ Notably,

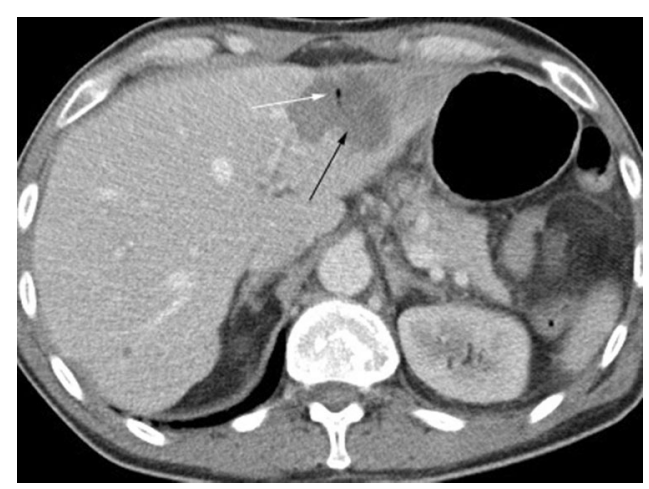

Fig. 1. Gas bubble in ablation zone. A CT obtained 1 week following microwave ablation demonstrates an elongated gas bubble (white arrow) in the center of the ablation zone (black arrow). In this early time-period such a finding is related to the ablation, not infection. 


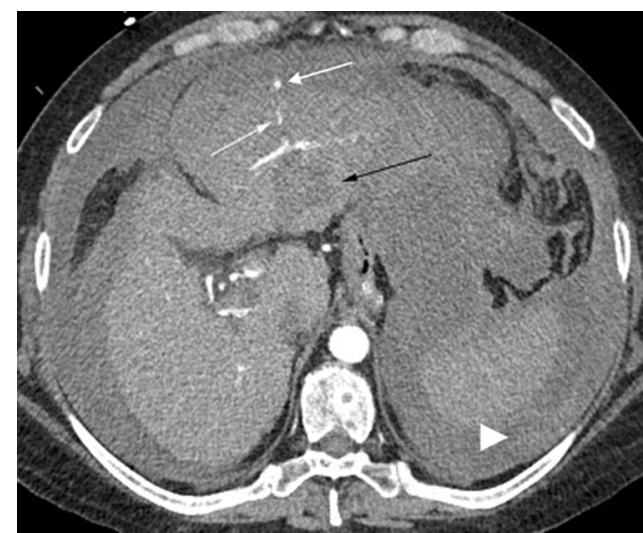

Fig. 2. Active hemorrhage. A CT in the arterial phase shortly after HCC ablation demonstrates that active arterial extravasation (white arrows) is present, emanating anteriorly from the ablation site (black arrow). The peripheral crescent of higher attenuation (arrowhead) suggests clotted blood along with the diffuse high attenuation peritoneal blood.

if an ablation zone abuts a blood vessel $>3 \mathrm{~mm}$ in diameter, it is common for residual disease or incomplete ablation to occur in this area. ${ }^{41,42}$ This phenomenon results from a "heat sink" effect, where the moving blood in the vessel cools the surrounding tissue, preventing lethal temperatures to be reached.

Therefore, on follow-up imaging, it is important to closely evaluate any areas adjacent to a large vessel. A final region to closely assess is the probe track. Track seeding is rare, occurring in only $0.2 \%$ of cases according to one large meta-analysis. ${ }^{43}$ Careful evaluation of the ablation track is still necessary so that this rare complication is not missed.

The timing of postablation imaging can vary between institutions. At the authors' institution, imaging is typically performed at 1 month, 3 months, and then every 3-6 months following treatment. Other imaging schedules have been suggested based upon risk stratification, but have not gained widespread acceptance. ${ }^{44}$ The authors' institution prefers contrast-enhanced MRI over contrast-enhanced CT, based primarily on the demonstrated superiority MRI has shown in diagnosing $\mathrm{HCC}^{28}$ The literature has mixed views of postablation imaging between MRI versus CT. Admittedly, the literature comparing these two modalities are older and therefore do not take into account the advances in both techniques. Some of the reports give MRI the advantage of visualizing residual/recurrent disease, while another paper suggests MRI and CT are equivalent in this task. ${ }^{45,46}$

\section{TACE}

\section{Technique}

TACE is commonly performed in two ways. The first, conventional TACE (CTACE) uses lipiodol, an oily radiopaque material, mixed with one or more chemotherapeutic agents followed by embolic particles to treat HCC. ${ }^{47}$ The second, drug-eluting bead TACE (DEB TACE) treats HCC with particles that slowly release a chemotherapeutic agent. ${ }^{47}$ Both techniques involve cannulation of the arteries feeding the tumor with a catheter or microcatheter and the delivery of one of the above described agents.

The goal of these techniques is to deliver a high dose of local chemotherapeutic agent, then stop blood supply to the tumor.
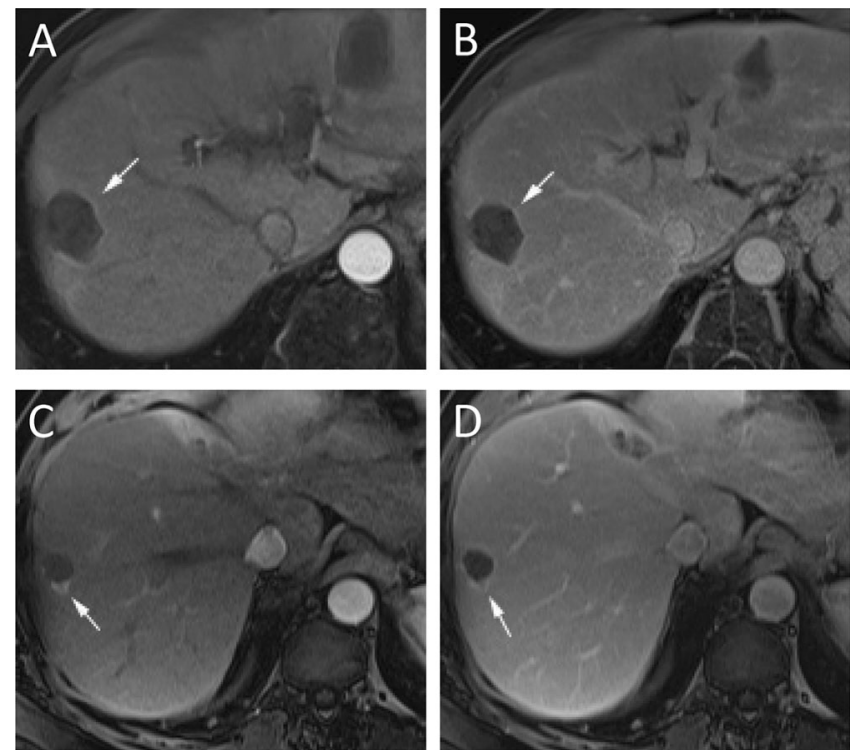

Fig. 3. PostMWA treatment changes. No residual tumor ( $A$ and $B$ ) and recurrent nodule of disease ( $C$ and $D)$. (A) Arterial phase imaging, 1 month after MWA demonstrates a thin $(<5 \mathrm{~mm}$ ) rim (arrow) of enhancement without nodularity. (B) The thin rim of enhancement (arrow) persists on portal venous phase imaging. These findings are consistent with expected post treatment changes and not recurrence. (C) In another patient, arterial phase imaging shows a nodular area of enhancement (arrow) at the edge of the ablation zone. (D) This nodular lesion (arrow) demonstrates subtle washout on portal venous imaging.

Abbreviation: MWA, microwave ablation.

The goal of eliminating blood flow to the tumor is to both cause ischemic cell death and to increase the dwell time of the chemotherapeutic agent. A third technique in this LRT arm is TAE. TAE differs from both CTACE and DEB-TACE in that it simply treats the HCC with embolization alone, forgoing any chemotherapy. ${ }^{47}$ Multiple studies comparing these techniques have failed to show any significant difference in outcome; however, despite this, TAE is seldom used. ${ }^{48-50}$

TACE is contraindicated in patients with decompensated liver cirrhosis and bilobar extensive tumor involvement. ${ }^{51}$ Relative contraindications relate to other compromised organ function, especially related to the heart or lungs, HCC $>10 \mathrm{~cm}$ in diameter, untreated varices associated with variceal bleeding, and significant portal vein thrombus. ${ }^{51}$

\section{Imaging after TACE}

The differences in the previously described methods become critical during follow-up imaging. Lipiodol stains the treatment area/tumor for months following CTACE, while the contrast given with DEB-TACE and TAE typically washes out after a few hours. ${ }^{40,52}$ After cTACE, patients are typically imaged with a noncontrast CT within 24 hours to evaluate lipiodol distribution. The presence of lipiodol being taken up in the entire tumor is associated with complete necrosis. ${ }^{53}$ If the tumor does not show lipiodol coverage, the patient can be scheduled for a repeat TACE without having to wait for delayed follow-up imaging. If only a portion of the lesion is stained, it is not likely that the lesion will achieve complete response, and alternative-feeding arteries should be sought. ${ }^{54}$ It is common for HCC lesions to require more than one TACE to achieve maximal response. ${ }^{55}$

Similar to ablation, postTACE results are best evaluated on contrast-enhanced CT or MRI. As with ablation, lack of 
enhancement is a surrogate for tumor death, and has been found to correlate well with pathologic necrosis. ${ }^{54}$ Following TACE, as with ablation, it is not uncommon to see a thin rim of enhancement around the treated lesion. If this rim is $\leq 5 \mathrm{~mm}$ in width and shows persistent enhancement, not washout, on portal venous phase imaging, it most often does not signify recurrence (Fig. $4 \mathrm{~A}$ and $4 \mathrm{~B}$ ). ${ }^{40}$ Residual tumor is primarily demarcated by nodular, usually $>5 \mathrm{~mm}$, areas that demonstrate arterial enhancement and subsequent washout. Recurrences are most commonly at the edge of the treatment area (Fig. 4C and 4D). ${ }^{56}$

Again, timing for posttreatment follow-up with imaging is at 1 month, 3 months, and then every 3-6 months at the authors' institution. Similar to ablation, alternative follow-up schedules have been suggested, but have not attained general acceptance. ${ }^{44} \mathrm{~A}$ recent survey of Society of Interventional Radiology (SIR) members found that nearly two-thirds of responders preferred 1 month follow-up posttreatment for conventional TACE, DEB-TACE, and TAE at 63\% (54/86), 64\% (53/83), and $64 \%$ (50/78) respectively. ${ }^{57}$

The deposition of lipiodol can lead to beam hardening artifact and obscure enhancement on arterial phase, reducing the sensitivity of CT for postcTACE imaging. ${ }^{26,58}$ Recent advances in dual-energy CT may allow the sensitivity of contrast-enhanced CT to be increased following CTACE, but this technology is not readily available for clinical use. ${ }^{59,60}$ The authors' institution prefers the use of MRI given the reduced sensitivity seen from any lipiodol artifact and the
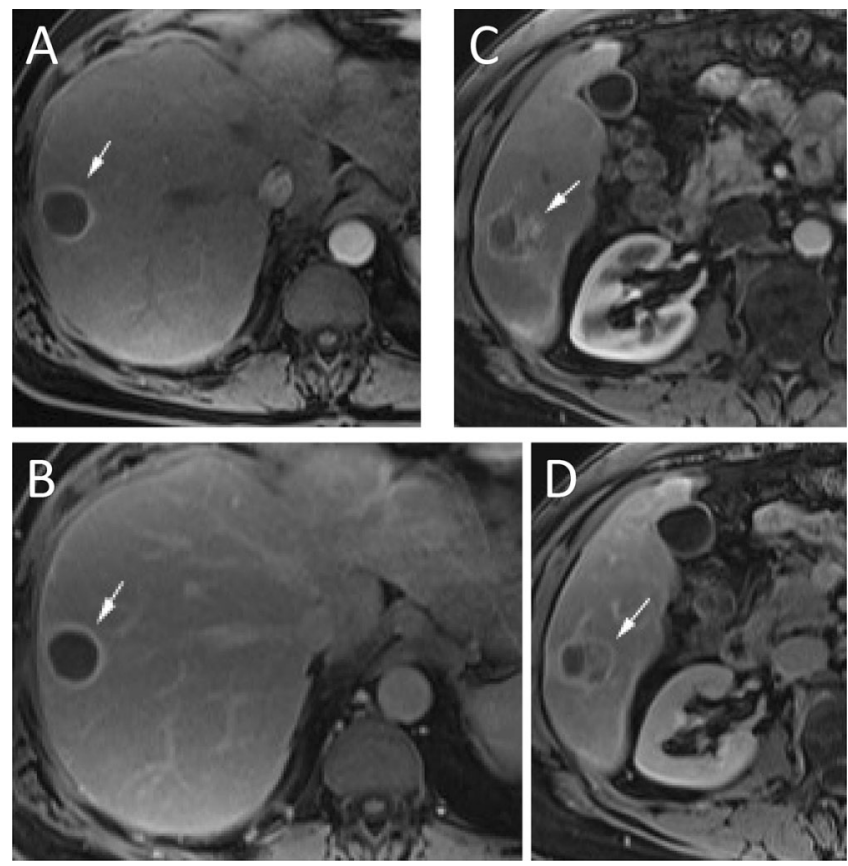

Fig. 4. PostTACE treatment. No residual tumor ( $A$ and $B$ ) and residual disease present ( $C$ and $D)$. (A) Arterial phase imaging, 1 month after TACE demonstrates a thin $(<5 \mathrm{~mm}$ ) rim (arrow) of enhancement without nodularity. (B) The thin rim of enhancement (arrow) persists on portal venous phase imaging. These findings are consistent with expected post treatment changes and not recurrence. (C) In another patient, arterial phase imaging, 1 month after TACE demonstrates a nodular $(>5 \mathrm{~mm}$ ) area of enhancement (arrow). (D) This nodular area of enhancement (arrow) demonstrates washout on portal venous phase imaging. These findings are consistent with recurrent $\mathrm{HCC}$.

Abbreviations: HCC, hepatocellular carcinoma; TACE, transarterial chemoembolization. previously mentioned superior performance of MRI in the pretreatment phase. $26,28,58$

\section{TARE}

\section{Technique}

TARE is similar to TACE in that it involves treatment of tumors by delivering particles to the artery feeding the tumor through a microcatheter. However, TARE differs from the other techniques in that these particles are radioactive and induce tumor death through beta-radiation. ${ }^{61}$ Furthermore, while TARE is delivered via arterial injection, it is not considered to be embolic, but microembolic. ${ }^{61}$ This is starkly different from TACE, which relies on a second step of embolization to stop blood flow to the tumor and induce tumor death. TARE does not induce tumor death by stopping blood flow but rather by beta radiation. This radiation effect is potentiated by the oxygen delivered by continued blood flow, and thus the persistent blood flow to the region is beneficial.

Contraindications to TARE are the presence of a pretreatment testing shunt to the lungs that would potentially deliver $>30$ Gy radiation pulmonary exposure, or potential gastrointestinal embolism. Relative contraindications relate to poor hepatic reserve, irreversible elevated bilirubin, and prior hepatic radiation. 62

\section{Imaging after TARE}

The beta radiation emitted by the intraarterially delivered yttrium 90 causes diffuse increase in blood flow to the treated area that translates to increased contrast enhancement on
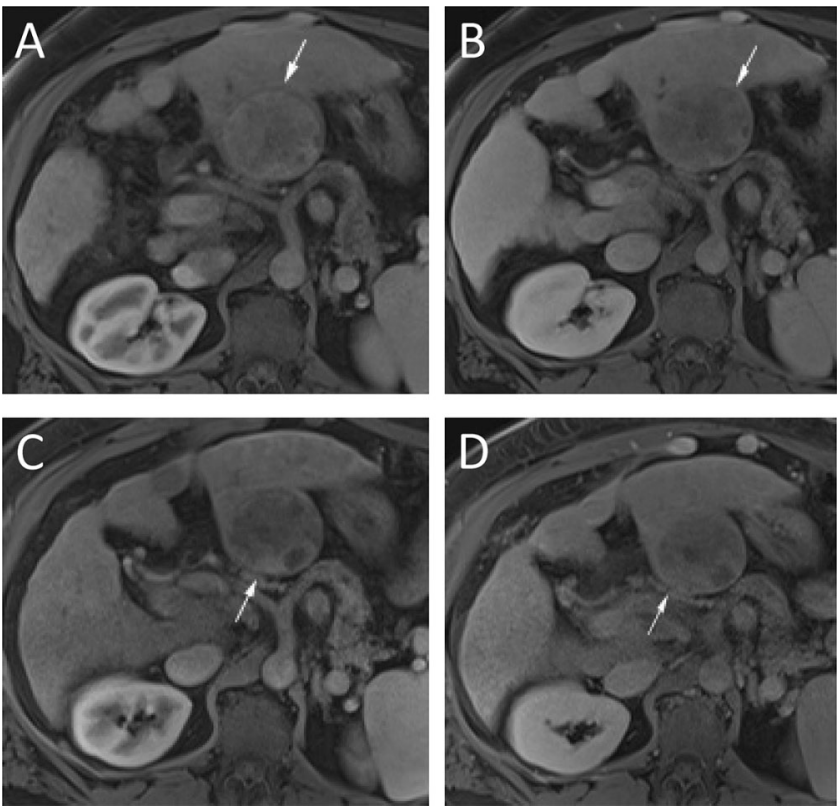

Fig. 5. Transarterial radioembolization treatment imaging findings. One month imaging shows diffuse patchy enhancement on arterial $(A)$ and, to a lesser extent, on portal venous (B) phase imaging (arrows). While multiple areas of patchy enhancement are present at 1 month, the 3 months imaging shows an area of enhancement on arterial (C) and washout on portal venous (D) imaging (arrows). Because of these MRI findings, this lesion was considered residual disease and treated again without histologic confirmation. 

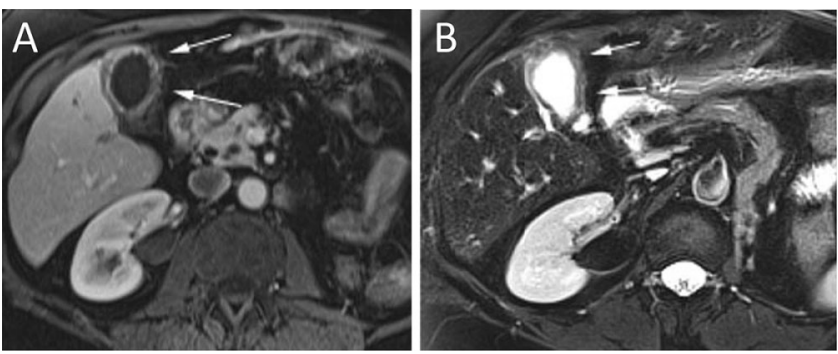

Fig. 6. Complication of TARE, nontarget embolization. MRI following TARE demonstrates diffuse gallbladder wall thickening on postcontrast $(A)$ and T2-weighted (B) images (arrows) for radiation cholecystitis.

Abbreviation: TARE, transarterial radioembolization.

follow-up imaging, termed "radiation effect". ${ }^{22,63}$ This diffuse enhancement pattern can frequently lead to confusion over the presence or absence of residual disease. ${ }^{18,64}$ It is common for patients to develop patchy arterial enhancement throughout the treatment zone, which is poorly predictive of residual disease prior to 90 days posttreatment (Fig. 5A and 5B). ${ }^{18,64}$

After 90 days, this patchy enhancement dissipates and lesions that show typical characteristics of HCC, namely LAP enhancement with PVP/DP washout (Fig. 5C and 5D), can be more reliably defined as such. After 90 days posttreatment, as with other treatment methods, it is expected that successfully treated areas will show a lack of arterial enhancement. It should be noted that a variable amount of adjacent "normal" liver will be included in the treatment field. It is common that with time this liver will slowly retract and become less prominent along with the treated tumor. Frequently, fibrosis will develop in this "normal" liver and show the typical fibrotic enhancement pattern of increasing enhancement on PVP/DP. Also on followup, imaging can display evidence of the rare instance of "nontarget" embolization with its radiation effects (Fig. 6).

Timing of posttreatment imaging is somewhat controversial in TARE, with many practitioners abstaining from imaging at
1 month, due to the enhancement issues discussed above. This was noted in a recent survey of SIR members, which found that imaging follow-up after TARE was preferred at 1 month by 26 of $74(35 \%)$ responders, while 32 of $74(43 \%)$ preferred the first imaging to be obtained at 3 months. ${ }^{57}$ These results differ from the other posttreatment timing reviewed above. The authors' institution again prefers MRI to CT for postTARE imaging, largely for the same reasons described in prior sections.

\section{Special considerations and evolving areas in} posttreatment imaging

\section{DWI}

DWI is a term used to refer to the restriction of water diffusion in tissue, as evaluated by two or more sequences on MRI. Water restriction is seen as hyperintense signal intensity on DWI sequences and hypointense signal intensity on apparent diffusion coefficient (ADC) sequences. Many tumors, including HCC, show restriction of water movement and are thus visible on DWI. Following treatment-induced necrosis, water restriction is "improved" (that is, the molecules can move around more freely) and this restriction is no longer present.

This microscopic change is reflected by an increase in the ADC signal intensity. ${ }^{65,66}$ This increase in ADC signal following treatment has been shown to correspond to necrosis. ${ }^{67}$ The DWI technique can be helpful both as another piece of imaging information in equivocal cases as well as when contrast enhancement is contraindicated. However, there has been a report that even with continued presence of restriction displayed on DWI, there may be pathologic necrosis. ${ }^{68}$

DWI has been used to try to help in the assessment of residual/recurrent disease within the first 90 days following TARE, in view of the problems related to contrast enhancement described above. Some authors have suggested that DWI can predict tumor response at 1 month (Fig. 7). ${ }^{69,70}$
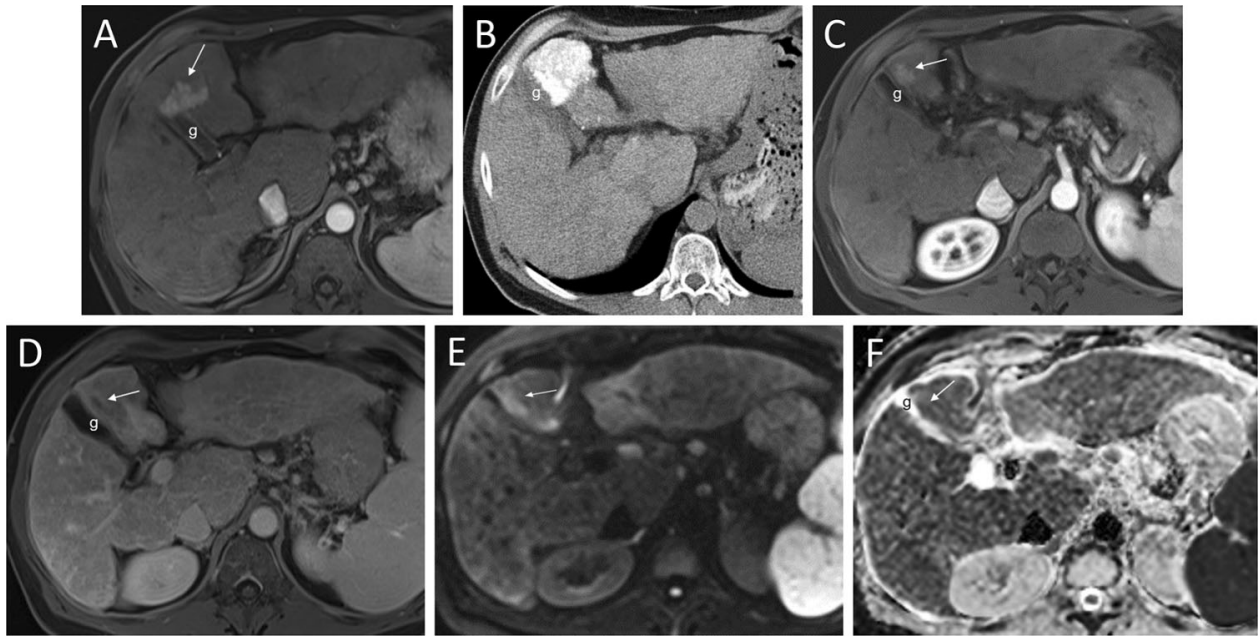

Fig. 7. DWI to help diagnosis post TACE residual. (A) Prior to treatment, an early arterial phase MRI demonstrates a hyperenhancing lobulated mass (arrow) in segment 4. Delayed phase washout and DWI sequences (not shown) established the imaging diagnosis of HCC. (B) Six weeks later this patient underwent conventional TACE. The CT following this procedure shows complete saturation of the HCC area with lipiodol. (C) Late arterial phase MRI one month later shows a continued hyperenhanced area (arrow). (D) A 3-minute delay image demonstrates washout (arrow). (E, F) The DWI series with correlating b 600 (E) and ADC (F) sequences confirm this area as residual HCC with high SI and low SI respectively (arrows). " $\mathrm{g}$ " marks the gallbladder.

Abbreviations: ADC, apparent diffusion coefficient; CT, computed tomography; DWI, diffusion weighted imaging; HCC, hepatocellular carcinoma; MRI, magnetic resonance imaging. 
Others, however, have not been able to reproduce DWI's value in this clinical scenario. ${ }^{71,72}$ While this area continues to be of interest, the variety of DWI techniques and disparity in results suggest it is not ready for clinical use in this setting ${ }^{72}$ and its importance remains to be determined.

\section{Perfusion imaging}

Attempts to determine tumor blood flow, referred to as "perfusion", have been made in both CT and MRI. CT attempts to estimate tumor perfusion by using single level imaging repetition after contrast administration, while MRI uses acquisition data from dynamic contrast-enhanced imaging. ${ }^{53}$ These techniques are used to create different parameters, such as "time to peak" and "mean transit time", among others. ${ }^{73}$ While some initial results have been promising, these techniques require dedicated software to perform the necessary calculations and there has been evidence of significant inconsistencies between software packages. ${ }^{73,74}$ The extra time, added costs, and relatively experimental nature of perfusion means that it is not currently in widespread use.

\section{Conclusions}

The imaging of HCC following locoregional therapies is of crucial clinical significance to the patient and the patient's multidisciplinary treatment team. While interpretation of the imaging can be challenging at times, multiple studies have elucidated key characteristics to help guide patient treatment decisions and accurately define treatment results.

\section{Conflict of interest}

The authors have no conflict of interests related to this publication.

\section{Author contributions}

Involved in the conception of the paper idea and critical revision of the paper and provided final approval for the article and the subsequent revised article (SY, AJT, TS), primarily responsible for article drafting (SY) and assistance (AJT, TS).

\section{References}

[1] Bosetti C, Turati F, La Vecchia C. Hepatocellular carcinoma epidemiology. Best Pract Res Clin Gastroenterol 2014;28:753-770. doi: 10.1016/j.bpg.2014. 08.007.

[2] Jadlowiec CC, Taner T. Liver transplantation: Current status and challenges. World J Gastroenterol 2016;22:4438-4445. doi: 10.3748/wjg.v22.i18.4438.

[3] Yao FY, Bass NM, Nikolai B, Davern TJ, Kerlan R, Wu V, et al. Liver transplantation for hepatocellular carcinoma: analysis of survival according to the intention-to-treat principle and dropout from the waiting list. Liver Transpl 2002;8:873-883. doi: 10.1053/jlts.2002.34923.

[4] Yegin EG, Oymaci E, Karatay E, Coker A. Progress in surgical and nonsurgical approaches for hepatocellular carcinoma treatment. Hepatobiliary Pancreat Dis Int 2016;15:234-256. doi: 10.1016/S1499-3872(16)60097-8.

[5] Cha C, Fong Y, Jarnagin WR, Blumgart LH, DeMatteo RP. Predictors and patterns of recurrence after resection of hepatocellular carcinoma. J Am Coll Surg 2003;197:753-758. doi: 10.1016/j.jamcollsurg.2003.07.003.

[6] Llovet JM, Ricci S, Mazzaferro V, Hilgard P, Gane E, Blanc JF, et al. Sorafenib in advanced hepatocellular carcinoma. N Engl J Med 2008;359:378-390. doi: 10.1056/NEJMoa0708857.

[7] Cheng AL, Kang YK, Chen Z, Tsao CJ, Qin S, Kim JS, et al. Efficacy and safety of sorafenib in patients in the Asia-Pacific region with advanced hepatocellular carcinoma: a phase III randomised, double-blind, placebo-controlled trial. Lancet Oncol 2009;10:25-34. doi: 10.1016/S1470-2045(08)70285-7.

[8] Lü MD, Kuang M, Liang LJ, Xie XY, Peng BG, Liu G], et al. Surgical resection versus percutaneous thermal ablation for early-stage hepatocellular carcinoma: a randomized clinical trial. Zhonghua Yi Xue Za Zhi 2006;86:801-805.

[9] Fang Y, Chen W, Liang X, Li D, Lou H, Chen R, et al. Comparison of long-term effectiveness and complications of radiofrequency ablation with hepatectomy for small hepatocellular carcinoma. J Gastroenterol Hepatol 2014;29: 193-200. doi: 10.1111/jgh.12441.

[10] Arslanoglu A, Seyal AR, Sodagari F, Sahin A, Miller FH, Salem R, et al. Current guidelines for the diagnosis and management of hepatocellular carcinoma: a comparative review. AJR Am J Roentgenol 2016;207:W88-W98. doi: 10. 2214/AJR.15.15490.

[11] Wald C, Russo MW, Heimbach JK, Hussain HK, Pomfret EA, Bruix J. New OPTN/UNOS policy for liver transplant allocation: standardization of liver imaging, diagnosis, classification, and reporting of hepatocellular carcinoma. Radiology 2013;266:376-382. doi: 10.1148/radiol.12121698.

[12] Heimbach JK, Kulik LM, Finn RS, Sirlin CB, Abecassis MM, Roberts LR, et al. AASLD guidelines for the treatment of hepatocellular carcinoma. Hepatology 2018;67:358-380. doi: 10.1002/hep.29086.

[13] EASL-EORTC clinical practice guidelines: management of hepatocellular carcinoma. J. Hepatol 2012;56:908-943. doi: 10.1016/j.jhep.2011.12.001.

[14] Therasse P, Arbuck SG, Eisenhauer EA, Wanders J, Kaplan RS, Rubinstein L, et al. New guidelines to evaluate the response to treatment in solid tumors. European Organization for Research and Treatment of Cancer, National Cancer Institute of the United States, National Cancer Institute of Canada. J Natl Cancer Inst 2000;92:205-216. doi: 10.1093/jnci/92.3.205.

[15] Eisenhauer EA, Therasse P, Bogaerts ], Schwartz LH, Sargent D, Ford R, et al. New response evaluation criteria in solid tumours: revised RECIST guideline (version 1.1). Eur J Cancer 2009;45:228-247. doi: 10.1016/j.ejca.2008.10.026.

[16] World Health Organization. WHO handbook for reporting results of cancer treatment. Geneva: World Health Organization, 1979. Available from: http: //www.who.int/iris/handle/10665/37200.

[17] Lencioni R, Llovet JM. Modified RECIST (mRECIST) assessment for hepatocellular carcinoma. Semin Liver Dis 2010;30:52-60. doi: 10.1055/s-00301247132.

[18] Singh P, Anil G. Yttrium-90 radioembolization of liver tumors: what do the images tell us? Cancer Imaging 2014;13:645-657. doi: 10.1102/14707330.2013.0057.

[19] Corona-Villalobos CP, Zhang $Y$, Zhang WD, Kamel IR. Magnetic resonance imaging of the liver after loco-regional and systemic therapy. Magn Reson Imaging Clin N Am 2014;22:353-372. doi: 10.1016/j.mric.2014.04.008.

[20] Kim JW, Seong J, Park MS, Kim KS, Park YN, Han KH, et al. Radiologicalpathological correlation study of hepatocellular carcinoma undergoing local chemoradiotherapy and surgery. J Gastroenterol Hepatol 2016;31: 1619-1627. doi: 10.1111/jgh.13334

[21] Jung ES, Kim JH, Yoon EL, Lee HJ, Lee S], Suh SJ, et al. Comparison of the methods for tumor response assessment in patients with hepatocellular carcinoma undergoing transarterial chemoembolization. J Hepatol 2013;58: 1181-1187. doi: 10.1016/j.jhep.2013.01.039.

[22] Yaghmai V, Besa C, Kim E, Gatlin JL, Siddiqui NA, Taouli B. Imaging assessment of hepatocellular carcinoma response to locoregional and systemic therapy. AJR Am J Roentgenol 2013;201:80-96. doi: 10.2214/AJR.13.10706.

[23] Chapiro J, Wood LD, Lin M, Duran R, Cornish T, Lesage D, et al. Radiologicpathologic analysis of contrast-enhanced and diffusion-weighted MR imaging in patients with HCC after TACE: diagnostic accuracy of 3D quantitative image analysis. Radiology 2014;273:746-758. doi: 10.1148/radiol.14140033.

[24] Chockalingam A, Duran R, Sohn JH, Schernthaner R, Chapiro J, Lee H, et al. Radiologic-pathologic analysis of quantitative 3D tumour enhancement on contrast-enhanced MR imaging: a study of ROI placement. Eur Radiol 2016;26:103-113. doi: 10.1007/s00330-015-3812-2.

[25] Stroehl YW, Letzen BS, van Breugel JM, Geschwind JF, Chapiro J. Intraarterial therapies for liver cancer: assessing tumor response. Expert Rev Anticancer Ther 2017;17:119-127. doi: 10.1080/14737140.2017.1273775.

[26] Kubota K, Hisa N, Nishikawa T, Fujiwara Y, Murata Y, Itoh S, et al. Evaluation of hepatocellular carcinoma after treatment with transcatheter arterial chemoembolization: comparison of Lipiodol-CT, power Doppler sonography, and dynamic MRI. Abdom Imaging 2001;26:184-190. doi: 10.1007/s002610000139.

[27] Westwood M, Joore M, Grutters J, Redekop K, Armstrong N, Lee K, et al. Contrast-enhanced ultrasound using SonoVue ${ }^{\circledR}$ (sulphur hexafluoride microbubbles) compared with contrast-enhanced computed tomography and contrast-enhanced magnetic resonance imaging for the characterisation of focal liver lesions and detection of liver metastases: a systematic review and cost-effectiveness analysis. Health Technol Assess 2013;17:1-243. doi: $10.3310 /$ hta17160

[28] Rostambeigi N, Taylor AJ, Golzarian J, Jensen EH, Pruett TL, Dudeja V, et al. Effect of MRI versus MDCT on milan criteria scores and liver transplantation eligibility. AJR Am J Roentgenol 2016;206:726-733. doi: 10.2214/AJR.15. 14642. 
[29] Braga L, Guller U, Semelka RC. Pre-, peri-, and posttreatment imaging of liver lesions. Radiol Clin North Am 2005;43:915-927. doi: 10.1016/j.rcl.2005.05. 005.

[30] Limanond P, Zimmerman P, Raman SS, Kadell BM, Lu DS. Interpretation of CT and MRI after radiofrequency ablation of hepatic malignancies. AJR Am J Roentgenol 2003;181:1635-1640. doi: 10.2214/ajr.181.6.1811635.

[31] Gravante G, Sconocchia G, Ong SL, Dennison AR, Lloyd DM. Immunoregulatory effects of liver ablation therapies for the treatment of primary and metastatic liver malignancies. Liver Int 2009;29:18-24. doi: 10.1111/j. 1478-3231.2008.01915.x.

[32] Sutter O, Calvo J, N'Kontchou G, Nault JC, Ourabia R, Nahon P, et al. Safety and efficacy of irreversible electroporation for the treatment of hepatocellular carcinoma not amenable to thermal ablation techniques: a retrospective single-center case series. Radiology 2017;284:877-886. doi: 10.1148/radiol. 2017161413.

[33] Luo W, Zhang Y, He G, Yu M, Zheng M, Liu L, et al. Effects of radiofrequency ablation versus other ablating techniques on hepatocellular carcinomas: a systematic review and meta-analysis. World J Surg Oncol 2017;15:126. doi: 10.1186/s12957-017-1196-2.

[34] Goldberg SN, Gazelle GS, Compton CC, Mueller PR, Tanabe KK. Treatment of intrahepatic malignancy with radiofrequency ablation: radiologic-pathologic correlation. Cancer 2000;88:2452-2463.

[35] Park MJ, Kim TH, Lee KM, Cheong JY, Kim JK. Radiofrequency ablation of metastatic liver masses: recurrence patterns and prognostic factors based on radiologic features. Hepatogastroenterology 2013;60:563-567. doi: 10. $5754 /$ hge12674.

[36] Suzuki C, Jacobsson H, Hatschek T, Torkzad MR, Bodén K, Eriksson-Alm Y, et al. Radiologic measurements of tumor response to treatment: practical approaches and limitations. Radiographics 2008;28:329-344. doi: 10.1148/ rg.282075068.

[37] Yaghmai V, Miller FH, Rezai P, Benson AB 3rd, Salem R. Response to treatment series: part 2, tumor response assessment-using new and conventional criteria. AJR Am J Roentgenol 2011;197:18-27. doi: 10.2214/AJR.11.6581.

[38] Smith S, Gillams A. Imaging appearances following thermal ablation. Clin Radiol 2008;63:1-11. doi: 10.1016/j.crad.2007.06.002.

[39] Chopra S, Dodd GD 3rd, Chintapalli KN, Leyendecker JR, Karahan OI, Rhim $H$. Tumor recurrence after radiofrequency thermal ablation of hepatic tumors: spectrum of findings on dual-phase contrast-enhanced CT. AJR Am J Roentgenol 2001;177:381-387. doi: 10.2214/ajr.177.2.1770381.

[40] Brennan IM, Ahmed M. Imaging features following transarterial chemoembolization and radiofrequency ablation of hepatocellular carcinoma. Semin Ultrasound CT MR 2013;34:336-351. doi: 10.1053/j.sult.2013.04.004.

[41] Lu DS, Raman SS, Vodopich DJ, Wang M, Sayre J, Lassman C. Effect of vessel size on creation of hepatic radiofrequency lesions in pigs: assessment of the "heat sink" effect. AJR Am J Roentgenol 2002;178:47-51. doi: 10.2214/ajr. 178.1.1780047.

[42] Lu DS, Raman SS, Limanond P, Aziz D, Economou J, Busuttil R, et al. Influence of large peritumoral vessels on outcome of radiofrequency ablation of liver tumors. J Vasc Interv Radiol 2003;14:1267-1274. doi: 10.1097/01. RVI.0000092666.72261.6B.

[43] Mulier S, Mulier P, Ni Y, Miao Y, Dupas B, Marchal G, et al. Complications of radiofrequency coagulation of liver tumours. Br J Surg 2002;89:1206-1222. doi: $10.1046 / j .1365-2168.2002 .02168 . x$

[44] Boas FE, Do B, Louie JD, Kothary N, Hwang GL, Kuo WT, et al. Optimal imaging surveillance schedules after liver-directed therapy for hepatocellular carcinoma. J Vasc Interv Radiol 2015;26:69-73. doi: 10.1016/j.jvir.2014. 09.013.

[45] Dromain C, de Baere T, Elias D, Kuoch V, Ducreux M, Boige V, et al. Hepatic tumors treated with percutaneous radio-frequency ablation: CT and MR imaging follow-up. Radiology 2002;223:255-262. doi: 10.1148/radiol.2231010780.

[46] Sironi S, Livraghi T, Meloni F, De Cobelli F, Ferrero C, Del Maschio A. Small hepatocellular carcinoma treated with percutaneous RF ablation: MR imaging follow-up. AJR Am J Roentgenol 1999;173:1225-1229. doi: 10.2214/ajr. 173.5.10541093.

[47] Pesapane F, Nezami N, Patella F, Geschwind JF. New concepts in embolotherapy of HCC. Med Oncol 2017;34:58. doi: 10.1007/s12032-017-0917-2.

[48] Llovet JM, Real MI, Montaña X, Planas R, Coll S, Aponte J, et al. Arterial embolisation or chemoembolisation versus symptomatic treatment in patients with unresectable hepatocellular carcinoma: a randomised controlled trial. Lancet 2002;359:1734-1739. doi: 10.1016/S0140-6736(02) 08649-X.

[49] Brown KT, Do RK, Gonen M, Covey AM, Getrajdman GI, Sofocleous CT, et al. Randomized trial of hepatic artery embolization for hepatocellular carcinoma using doxorubicin-eluting microspheres compared with embolization with microspheres alone. J Clin Oncol 2016;34:2046-2053. doi: 10.1200/JCO. 2015.64.0821.

[50] Lammer ], Malagari K, Vogl T, Pilleul F, Denys A, Watkinson A, et al. Prospective randomized study of doxorubicin-eluting-bead embolization in the treatment of hepatocellular carcinoma: results of the PRECISION V study.
Cardiovasc Intervent Radiol 2010;33:41-52. doi: 10.1007/s00270-0099711-7.

[51] Kumar Y, Sharma P, Bhatt N, Hooda K. Transarterial therapies for hepatocellular carcinoma: a comprehensive review with current updates and future directions. Asian Pac J Cancer Prev 2016;17:473-478.

[52] Golowa YS, Cynamon J, Reinus JF, Kinkhabwala M, Abrams M, Jagust M, et al. Value of noncontrast CT immediately after transarterial chemoembolization of hepatocellular carcinoma with drug-eluting beads. J Vasc Interv Radiol 2012;23:1031-1035. doi: 10.1016/j.jvir.2012.04.020.

[53] Adam SZ, Miller FH. Imaging of the liver following interventional therapy for hepatic neoplasms. Radiol Clin North Am 2015;53:1061-1076. doi: 10. 1016/j.rcl.2015.05.009.

[54] Riaz A, Memon K, Miller FH, Nikolaidis P, Kulik LM, Lewandowski RJ, et al. Role of the EASL, RECIST, and WHO response guidelines alone or in combination for hepatocellular carcinoma: radiologic-pathologic correlation. J Hepatol 2011;54:695-704. doi: 10.1016/j.jhep.2010.10.004

[55] White JA, Redden DT, Bryant MK, Dorn D, Saddekni S, Abdel Aal AK, et al. Predictors of repeat transarterial chemoembolization in the treatment of hepatocellular carcinoma. HPB (Oxford) 2014;16:1095-1101. doi: 10. 1111/hpb.12313.

[56] Park W, Chung YH, Kim JA, Jin YJ, Lee D, Shim JH, et al. Recurrences of hepatocellular carcinoma following complete remission by transarterial chemoembolization or radiofrequency therapy: Focused on the recurrence patterns. Hepatol Res 2013;43:1304-1312. doi: 10.1111/hepr.12083.

[57] Gaba RC, Baerlocher MO, Nikolic B, Venkatesan AM, Lewandowski RJ. Clinical and imaging follow-up practices after transarterial therapy for primary and secondary hepatic malignancies: results of an online survey. Acad Radiol 2015;22:1510-1515. doi: 10.1016/j.acra.2015.08.016.

[58] Takayasu K, Arii S, Matsuo N, Yoshikawa M, Ryu M, Takasaki K, et al. Comparison of CT findings with resected specimens after chemoembolization with iodized oil for hepatocellular carcinoma. AJR Am J Roentgenol 2000; 175:699-704. doi: 10.2214/ajr.175.3.1750699.

[59] Altenbernd J, Heusner TA, Ringelstein A, Ladd SC, Forsting M, Antoch G. Dual-energy-CT of hypervascular liver lesions in patients with HCC: investigation of image quality and sensitivity. Eur Radiol 2011;21:738-743. doi: 10.1007/s00330-010-1964-7.

[60] Lee JM, Yoon JH, Joo I, Woo HS. Recent advances in CT and MR imaging for evaluation of hepatocellular carcinoma. Liver Cancer 2012;1:22-40. doi: 10. $1159 / 000339018$.

[61] Sangro B, Iñarrairaegui M, Bilbao JI. Radioembolization for hepatocellular carcinoma. J Hepatol 2012;56:464-473. doi: 10.1016/j.jhep.2011.07.012.

[62] Kennedy A, Nag S, Salem R, Murthy R, McEwan AJ, Nutting C, et al. Recommendations for radioembolization of hepatic malignancies using yttrium-90 microsphere brachytherapy: a consensus panel report from the radioembolization brachytherapy oncology consortium. Int J Radiat Oncol Biol Phys 2007;68:13-23. doi: 10.1016/j.ijrobp.2006.11.060.

[63] Ibrahim SM, Nikolaidis P, Miller FH, Lewandowski RJ, Ryu RK, Sato KT, et al. Radiologic findings following $\mathrm{Y} 90$ radioembolization for primary liver malignancies. Abdom Imaging 2009;34:566-581. doi: 10.1007/s00261-0089454-y.

[64] Bester L, Hobbins PG, Wang SC, Salem R. Imaging characteristics following 90yttrium microsphere treatment for unresectable liver cancer. J Med Imaging Radiat Oncol 2011;55:111-118. doi: 10.1111/j.1754-9485.2011. 02241.x.

[65] Guo Y, Yaghmai V, Salem R, Lewandowski RJ, Nikolaidis P, Larson AC, et al. Imaging tumor response following liver-directed intra-arterial therapy. Abdom Imaging 2013;38:1286-1299. doi: 10.1007/s00261-013-0017-5.

[66] Sahin H, Harman M, Cinar C, Bozkaya H, Parildar M, Elmas N. Evaluation of treatment response of chemoembolization in hepatocellular carcinoma with diffusion-weighted imaging on 3.0-T MR imaging. J Vasc Interv Radiol 2012; 23:241-247. doi: 10.1016/j.jvir.2011.08.030.

[67] Mannelli L, Kim S, Hajdu CH, Babb JS, Clark TW, Taouli B. Assessment of tumor necrosis of hepatocellular carcinoma after chemoembolization: diffusion-weighted and contrast-enhanced MRI with histopathologic correlation of the explanted liver. AJR Am J Roentgenol 2009;193:1044-1052. doi: $10.2214 /$ AJR.08.1461.

[68] Gowdra Halappa V, Corona-Villalobos CP, Bonekamp S, Li Z, Reyes D, Cosgrove $\mathrm{D}$, et al. Neuroendocrine liver metastasis treated by using intraarterial therapy: volumetric functional imaging biomarkers of early tumor response and survival. Radiology 2013;266:502-513. doi: 10.1148/radiol.12120495.

[69] Schmeel FC, Simon B, Sabet A, Luetkens JA, Träber F, Schmeel LC, et al. Diffusion-weighted magnetic resonance imaging predicts survival in patients with liver-predominant metastatic colorectal cancer shortly after selective internal radiation therapy. Eur Radiol 2017;27:966-975. doi: 10.1007/ s00330-016-4430-3.

[70] Vouche M, Kulik L, Atassi R, Memon K, Hickey R, Ganger D, et al. Radiologicalpathological analysis of WHO, RECIST, EASL, mRECIST and DWI: Imaging analysis from a prospective randomized trial of $Y 90 \pm$ sorafenib. Hepatology 2013;58:1655-1666. doi: 10.1002/hep.26487. 
[71] Duke E, Deng J, Ibrahim SM, Lewandowski RJ, Ryu RK, Sato KT, et al. Agreement between competing imaging measures of response of hepatocellular carcinoma to yttrium-90 radioembolization. J Vasc Interv Radiol 2010;21: 515-521. doi: 10.1016/j.jvir.2009.11.020.

[72] Ludwig JM, Camacho JC, Kokabi N, Xing M, Kim HS. The Role of diffusionweighted imaging (DWI) in locoregional therapy outcome prediction and response assessment for hepatocellular carcinoma (HCC): the new era of functional imaging biomarkers. Diagnostics (Basel) 2015;5:546-563. doi: 10.3390/diagnostics5040546.
[73] Chen X, Xiao $E$, Shu D, Yang $C$, Liang $B$, He Z, et al. Evaluating the therapeutic effect of hepatocellular carcinoma treated with transcatheter arterial chemoembolization by magnetic resonance perfusion imaging. Eur J Gastroenterol Hepatol 2014;26:109-113. doi: 10.1097/MEG. Ob013e328363716e.

[74] Gonzalez-Guindalini FD, Botelho MP, Harmath CB, Sandrasegaran K, Miller $\mathrm{FH}$, Salem R, et al. Assessment of liver tumor response to therapy: role of quantitative imaging. Radiographics 2013;33:1781-1800. doi: $10.1148 / \mathrm{rg}$. 336135511. 